\title{
The Effect of Ginger on Pain and Satisfaction of Patients with Knee Osteoarthritis
}

\author{
Zeinab Alipour, ${ }^{1}$ Marziyeh Asadizaker,, ${ }^{1, *}$ Sedigheh Fayazi, ${ }^{1}$ Nima Yegane, ${ }^{2}$ Marym Kochak, ${ }^{3}$ and \\ Mohammad Hossein Haghighi Zadeh ${ }^{4}$ \\ ${ }^{1}$ Nursing Care Research Center in Chronic Diseases, Nursing and Midwifery School, Ahvaz Jundishapur University of Medical Sciences, Ahvaz, IR Iran \\ ${ }^{2}$ Department of Orthopedic, University of Medical Sciences, Dezful, IR Iran \\ ${ }^{3}$ Department of Pharmaceutics, School of Pharmacy, Ahvaz Jundishapur University of Medical Sciences, Ahvaz, IR Iran \\ ${ }^{4}$ Department of Epidemiology and Statistics, Ahvaz Jundishapur University of Medical Sciences, Ahvaz, IR Iran \\ "Corresponding author: Marziyeh Asadizaker, Ahvaz Jundishapur University of Medical Sciences, Ahvaz, IR Iran. Tel: +98-9166159157, Fax: +98-6133733822, E-mail: \\ marziyehasadizaker@gmail.com
}

Received 2015 November 18; Revised 2016 January 11; Accepted 2016 February 03.

\begin{abstract}
Background: Knee osteoarthritis is the most common type of arthritis and musculoskeletal disease. Due to low efficacy and significant side effects of synthetic drugs in the treatment of this disease, many researchers are looking for drugs that besides effectiveness have fewer side effects, and recently ginger has become one of the most popular herbal remedies in the treatment of this disease in controversial researches.

Objectives: The aim of this study was to determine the effect of ginger on pain and satisfaction of patients with knee osteoarthritis. Methods: This study was a clinical trial. A total of 90 patients with knee osteoarthritis were randomly divided to ginger and control groups. In addition, treatment was prescribed by orthopedic specialists; the ginger group received two tablets of $500 \mathrm{mg} / \mathrm{day}$ for 12 weeks. The control group only received the treatment prescribed by the orthopedic specialist. Pain was measured using a linear visual assessment scale (VAS) and satisfaction using a researcher-made questionnaire at the beginning and end of the twelfth week. Results: There was no significant difference between pain scores of the two groups before the intervention, yet after the intervention, pain scores declined in both groups, but it was greater in the ginger group $(\mathrm{P}=0.001)$. In terms of satisfaction with treatment, a statistically significant difference was shown between the two groups $(\mathrm{P}=0.012)$, so that it was higher in the group receiving ginger. On the other hand, the incidence of side effects was not statistically significant between the two groups.

Conclusions: The results of this study showed that ginger is effective in relieving pain in patients with knee osteoarthritis and it can be used as a safe method to improve pain in patients with knee osteoarthritis.
\end{abstract}

Keywords: Knee Osteoarthritis, Pain, Ginger, Satisfaction, Side Effects

\section{Background}

Osteoarthritis also known as degenerative joint disease is the most common type of arthritis and musculoskeletal disease $(1,2)$ so that its prevalence is more than heart disease, high blood pressure and diabetes (3). It is the leading cause of disability in the elderly $(2,4)$, so that one- third of older people are affected by the disease (5). Because of aging of Western societies as well as an increase in the prevalence of obesity (a major risk factor), the incidence of osteoarthritis is increasing (6). This disease is also a major cause of pain and disability in most countries of the world (2, 7), and affects all age groups (3), so that it was prevalent in males before 45 years old and in femlaes at 55 years old and its prevalence increases with age in both genders (8). Also it is one of the important reasons for the decline in activities of daily living and low quality of life in older people (9), and has a significant impact on the lives of individuals, including mobility, independence and ev- eryday activities and leads to a restriction of recreational, sport and occupational activities (10).

The most common affected joint by osteoarthritis is the knee (11). Moreover, knee arthritis is the most common cause of chronic disability in the elderly in developed countries, and in 2005, it was found that about one hundred thousand people in America were affected by this condition, who were unable to get out of bed and do their own health care without help (6). Forty percent of people over 70 years have knee osteoarthritis around the world (12).

In epidemiological studies, the prevalence of knee osteoarthritis was $7.9 \%$ around the world, $5 \%$ in urban areas of India, $4 \%$ in rural areas, $1.8 \%$ in Pakistan, $1.3 \%$ in indigenous Australia, $15.3 \%$ in urban areas of Iran and $19.3 \%$ in rural areas of Iran. It has been reported that in Iran, the prevalence of this complication has been higher than other studied countries (13). In both industrialized and developing countries, osteoarthritis has been increasingly and con- 
sidered as an important cause of pain and disability, and because of certain habits and more pressure on the knee joint, osteoarthritis of the knee is common and appears at an earlier age in Iran (14).

Treatment of osteoarthritis includes nonpharmacological, medication-based, surgical and complementary treatment. There are some concerns that some of these treatments cannot be completely effective. Some trial data have shown that the use of new non-steroidal anti-inflammatory drugs (NSAIDs)) increased cardiovascular problems, and traditional types of these drugs cause gastrointestinal irritation $(15,16)$. In addition, in a study it was shown that non-steroidal anti-inflammatory drugs are usually prescribed to inhibit the synthesis of cartilage matrix in humans, which in turn increases cartilage damage in osteoarthritis joints (17). Therefore, many researchers are looking for drugs, which are both effective and have fewer side effects, among which we can mention the ginger plant (18-20).

Recently, ginger has become one of the most popular herbal remedies in rheumatologic diseases (21). The World Health Organization considered ginger as a food supplement (22). Ginger is used as a medicine, spice and food around the world (23). Also, it is listed in the Food and drug administration (FDA) as a medicine (24). The antiinflammatory effects of ginger were firstly documented in scientific literature in 1982, and they found that the anti-inflammatory effects of ginger are the result of constituents such as Prostaglandin (PG) and Leukotriene (LT) (25). Ginger belongs to the Zingiber Officinale family, and although it is usually at the root of the ginger plant, it is a swollen underground stem called a rhizome (26). Ginger rhizome is used for Ayurveda medicine and in Chinese and Japanese medicine for treatment of inflammation and arthritis for thousands of years; other uses include dizziness, nausea, vomiting caused by motion sickness, postoperative nausea and vomiting during pregnancy (27). Ginger contains hundreds of known substances including gingerols, beta-carotene, Kapsyaysyn, caffeic acid and curcumin (25). Several studies have shown that ginger has anti-inflammatory and analgesic effect (28) through inhibition of cyclooxygenase and lipoxygenase pathways and prevention of arachidonic acid metabolism (29).

\section{Objectives}

This study aimed to determine the effect of ginger on pain and satisfaction of patients with knee osteoarthritis.

\section{Methods}

This study is a clinical trial conducted from March to November in 2015. The participants of this study consisted of patients with knee osteoarthritis, who had refferred to Afshar clinic in Dezful in the north of Khuzestan province.

Inclusion criteria: age of 50 years or older; confirmed osteoarthritis by orthopedic specialist; lack of inflammatory diseases, metabolic disorders (diabetes), cancer or serious illness, schizophrenia, Alzheimer's disease and intraarticular injection at least for the past 6 months; and lack of regular doses of ginger. Refusing participation in the study and sensitivity to ginger were the exclusion criteria. Non-compliance with the study protocol and any side effects of ginger were a reason for drop-out.

The calculation of the sample size was determined considering error of $5 \%$, and $90 \%$ for each group of 40 patients; thus a total of 90 patients participated in the study based on the inclusion criteria. Eligible patients of the study were randomly assigned to either the ginger $(n=45)$ or the control $(n=45)$ groups. Both groups received conventional treatment of knee osteoarthritis prescribed by the orthopedic specialist. In addition, the ginger group received two tablets /day containing $500 \mathrm{mg}$ of ginger for 12 weeks (Naderi et al. 2012) (30).

Follow-up or examination of possible side effects and adherence to the study protocol was conducted through reference to Afshar clinic and by telephone contact every week. If a person took more than $90 \%$ of capsules, adherence was complete and it was evaluated by counting the number of remaining cylinders at the end of the 12 weeks.

At the beginning and end of the study, pain was assessed with the help of visual assessment scale (VAS). This measure is a 10-cm ruler by which the patient will be asked to rate their pain intensity from zero to 10.

Individuals must mark their pain pre-dose and at the end of the twelfth week. Scores of 0 to 3 indicated mild pain, 4 to 6 moderate, 7 to 9 severe and 10 was unbearable pain. At the beginning of the study, questionnaires of demographic characteristics including age, gender, marital status, education level and employment status, location and duration of illness involving the knees were completed.

The draft of the study was approved by the research ethics committee of Ahvaz University of Medical Sciences and Health Services, and was registered in the clinical trial site of the department of science and technology of the ministry of health. Written consent form was signed by the patients. No fee was paid by patients and patient data were retained. Patients were recruited willingly and they could stop participation at any point in the study. Only the data of patients, who completed the treatment were statis- 
tically analyzed. Statistical analysis of data was conducted using the SPSS software version 20.

\section{Results}

At the beginning, the study included 90 individuals, from each group three patients were excluded from the analysis because of digestive problems, two patients for travelling and three patients for failing to comply with the study protocol (incomplete use of supplements). Finally, 77 patients ( 40 controls and 37 subjects in the intervention group) were subject to statistical analysis (Figure 1).

At the beginning of the intervention, patients' demographic characteristics were not significantly different in terms of age, gender, level of education, place of residence and duration of disease, between the two groups ( $\mathrm{P}>0.05)$ (Table 1).

Table 1. Demographic Characteristics of the Participants

\begin{tabular}{|lccc|}
\hline Variable & Ginger & Control & P Value \\
\hline Age & $58.6 \pm 50$ & $57.7 \pm 68$ & 0.58 \\
\hline Duration of disease & $3.7 \pm 9.78$ & $2.7 \pm 9.1$ & 0.13 \\
\hline Gender & & & 0.28 \\
\hline Male & $29(78)$ & $28(70)$ & \\
\hline Female & $8(21.6)$ & $7(17.5)$ & 0.70 \\
\hline Education Level & & & \\
\hline High school & $30(81.1)$ & $28(70)$ & \\
\hline Diploma & $4(10.8)$ & $7(17.5)$ & 0.34 \\
\hline Bs and higher & $3(8.1)$ & $3(7.5)$ & \\
\hline Location & & & \\
\hline Urban & $31(83.8)$ & $31(77.5)$ & 0.07 \\
\hline Rural & $6(16.2)$ & $9(22.5)$ & \\
\hline Affected Knee & & $7(42.5)$ & \\
\hline Right & $6(16.2)$ & $12(30)$ & \\
\hline Left & & & \\
\hline Both & & & \\
\hline
\end{tabular}

Distribution of frequency severity of pain before and after treatment in both groups is shown in Table 2. As can be seen, before the intervention average pain scores between the two groups was not statistically significant $(\mathrm{P}=0.66)$, yet after the intervention, pain scores declined in both groups, but was greater in the ginger group $(\mathrm{P}=$ 0.001) (Table 2). The average pain score decreased in the control group. This reduction of pain can be due to drugs, as prescribed by the orthopedic specialist.
Table 2. Comparison of Severity of Mean Pain Before and After the Intervention in Both Groups

\begin{tabular}{lccc}
\hline Group & Before Intervention & After Intervention & PValue $^{\mathbf{b}}$ \\
\hline Ginger & $7.78 \pm 2.90$ & $2.18 \pm 2.59$ & 0.001 \\
Control & $7.50 \pm 2.72$ & $4.9 \pm 3.04$ & 0.001 \\
Pvalue $^{\mathbf{c}}$ & 0.66 & 0.001 & \\
\hline
\end{tabular}

${ }^{\mathrm{a}}$ Values are expressed as mean $\pm \mathrm{SD}$.

${ }^{\mathrm{b}}$ Wilcoxon.

${ }^{\mathrm{c}}$ Student t-test.

As shown in Table 3, the two groups regarding the incidence of adverse events were not significantly different $(P>0.05)$, yet there was a significant difference in type of side effects between them, so that gastrointestinal symptoms in the control group were higher than the ginger group and non-gastrointestinal symptoms (such as itching and hot flashes) were observed in three cases of the ginger group while non-GI complications were not observed in the control group.

Table 3. Comparison of the Two Groups in Terms of Side Effects and Satisfaction

\begin{tabular}{|cccc}
\hline Variable & Ginger & Control & P Value \\
\hline Side Effects & & & 0.79 \\
Yes & $11(29.7)$ & $13(32.5)$ & \\
\hline No & $26(70.3)$ & $27(67.5)$ & \\
Type of Side Effects & & & 0.04 \\
\hline Digestive & $8(21.6)$ & $13(32.5)$ & \\
\hline Non digestive & $3(8.1)$ & 0 & 0.012 \\
\hline Satisfaction & & & \\
\hline Yes & $33(89.2)$ & $26(65)$ & \\
\hline No & $4(10.8)$ & $14(35)$ & \\
\hline
\end{tabular}

As shown in Table 3, a statistically significant difference was shown between the two groups in terms of satisfaction with treatment $(P=0.012)$, so that satisfaction with treatment was higher in the group receiving ginger.

\section{Discussion}

The aim of this study was to determine the effect of ginger on pain and satisfaction of patients with knee osteoarthritis. This study showed that ginger can be used as a medicine and supplement, to reduce pain in patients with knee osteoarthritis. Several studies have reported such effects. In a study by Altman et al. that evaluated the analgesic effect of ginger extract on pain in patients with osteoarthritis of the knee in 2001, after the intervention, knee 


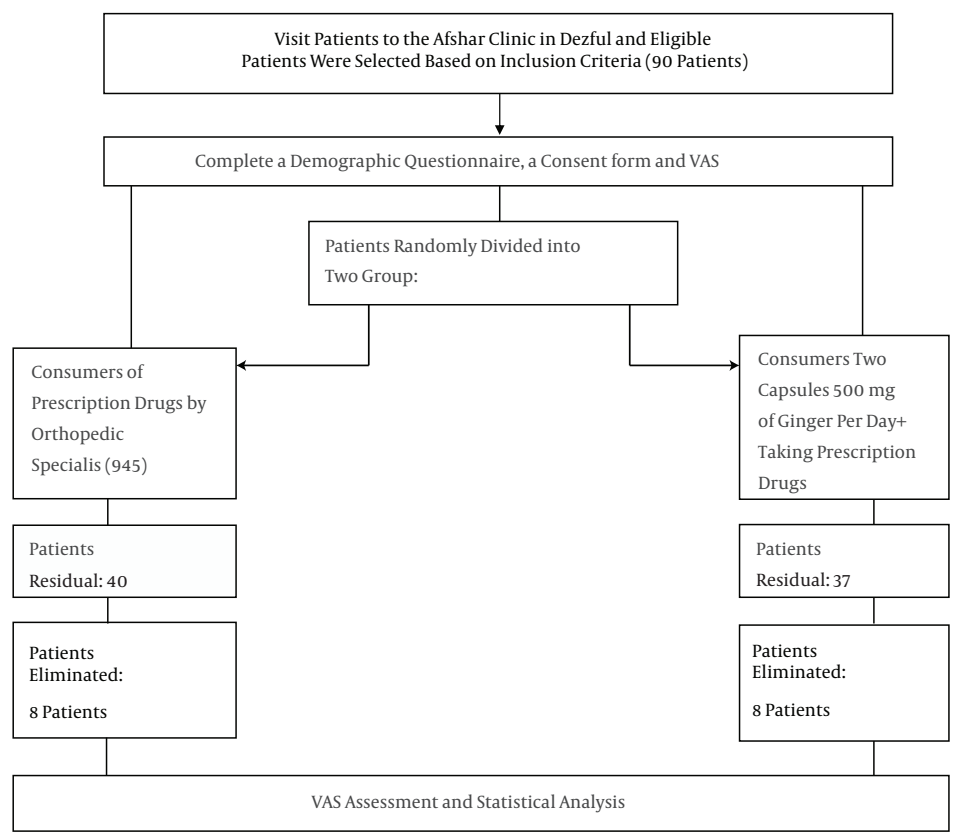

Figure 1. Flow Chart of the Steps Involved in this Study

pain in the ginger group was less than the placebo group and there was a significant difference in pain between the two groups before and after the intervention, so that the pain change decreased in both groups, yet this reduction was more significant in the ginger group (31). The findings of this study are consistent with the findings of the mentioned study despite more samples and shorter duration and lower ginger dose.

In 2005, in another study by Haghighi et al. the researchers compared the effect of ginger and ibuprofen in 120 patients with moderate to severe pain due to knee or hip osteoarthritis; the comparison of pain assessment and sample size in this study was similar to the present study, while treatment duration and dose of medication were less. However, the findings were consistent with the present findings (32).

In 2012, in another study by Naderi et al. the researchers aimed to determine the effect of ginger powder supplement on relief of pain in 120 patients with moderate knee osteoarthritis from Yazd city of Iran; the clinical trial was conducted in a double blind, placebo-controlled procedure, and pain intensity in both groups was not significantly different. However, no significant difference was achieved after the intervention, so that before the intervention, more than $90 \%$ of pain scores in both groups was between 4 and 7 while after the intervention $6 \%$ of the ginger group and $78 \%$ of placebo had these pain scores. On the other hand, the average change in pain scores after the intervention was significantly decreased in ginger and placebo groups were $88 \%$ and $14 \%$, respectively and there was a statistically significant difference between the two groups ( $\mathrm{P}<0.0001$ ) (30). The present study investigated a similarity is that in both capsules of powdered ginger has been used for 12 weeks. In our study, patients were excluded from the standard treatment and both groups received treatment prescribed by an orthopedic specialist. In this study, adverse events in terms of satisfaction with treatment was measured.

In a study by Srivastava et al. raw ginger at a dose of 3 grams per day for at least three months reduced pain and swelling of joints in patients with joint diseases (33). In a study by Sharma, Ramezani et al. similar results were obtained $(34,35)$.

In our study, three patients in the control group and three patients in the ginger group due to average gastrointestinal problems were not able to continue the project. However, gastrointestinal side effects were observed in 14 patients in the control group, (32.5\%) and digestive complications in the intervention group were observed in eight patients (21.8\%). In addition, three patients (1.8\%) had nongastrointestinal symptoms such as itching and a feeling of flushing (feeling of warmth). Such symptoms were observed in the control group. However, complications were mild and participants were able to finish the project. 
In the study of Altman et al. about $28 \%$ of patients treated with ginger and $11 \%$ of placebo-treated patients had withdrawn due to gastrointestinal problems (31). Also, in the study of Khalvat about 12 patients (16\%) in the ginger group and seven patients (3.9\%) treated with placebo were not primarily able to continue the project due to gastrointestinal problems (32).

The majority of the complications were caused by the consumption of ginger including indigestion, burning sensation behind the breastbone, bloating, nausea and bad taste in the mouth. According to Altman et al. 70\% of cases reported mild, $24 \%$ moderate, and $6 \%$ severe complications (31). In this study, complications were moderate so that participants were able to continue the study and only six patients (5.4\%) had unbearable gastrointestinal side effects.

This study is one of the few studies to examine the longterm effect of ginger on pain in patients with osteoarthritis. The strengths of this study was that it assessed satisfaction and side effects in patients. Further studies should be conducted on the effect of ginger on osteoarthritis pain in other joints as well as the effect of ginger on other clinical symptoms of knee osteoarthritis.

\subsection{Conclusions}

This study showed that one gram of ginger per day for 12 weeks can reduce pain in patients with knee osteoarthritis.

\section{Acknowledgments}

At the end, the authors thank all those, who helped us in conducting this study, especially personnel in Afshar hospital of Dezfoul, and all patients in this study, who accompanied us.

\section{Footnotes}

Authors' Conrtribution: Zeinab Alipour, Marziyeh Asadizaker, Sedigheh Fayazi and Marym Kochak were responsible for the study conception and design. Mohammad Hossein Haghighi Zadeh was responsible for the statistical analysis. Data collection was conducted by Zeinab Alipour and Nima Yegane. All authors approved the final manuscript.

Funding/Support: This article was part of the MSc thesis of the first author (Zeinab Alipour), and was financially supported by chronic disease care research center, department nursing, Ahvaz Jundishapur University of Medical Sciences.

\section{References}

1. Zhang W, Moskowitz RW, Nuki G, Abramson S, Altman RD, Arden $\mathrm{N}$, et al. OARSI recommendations for the management of hip and knee osteoarthritis, Part II: OARSI evidence-based, expert consensus guidelines. Osteoarthritis Cartilage. 2008;16(2):137-62. doi: 10.1016/j.joca.2007.12.013. [PubMed: 18279766].

2. Felson DT. Clinical practice. Osteoarthritis of the knee. N Engl J Med. 2006;354(8):841-8. doi: 10.1056/NEJMcp051726. [PubMed: 16495396].

3. Kelsey JL, Hochberg MC. Epidemiology of chronic musculoskeletal disorders. Annu Rev Public Health. 1988;9:379-401. doi: 10.1146/annurev.pu.09.050188.002115. [PubMed: 2967707].

4. Peat G, McCarney R, Croft P. Knee pain and osteoarthritis in older adults: a review of community burden and current use of primary health care. Ann Rheum Dis. 2001;60(2):91-7. [PubMed: 11156538].

5. Poitras S, Avouac J, Rossignol M, Avouac B, Cedraschi C, Nordin M, et al. A critical appraisal of guidelines for the management of knee osteoarthritis using Appraisal of Guidelines Research and Evaluation criteria. Arthritis Res Ther. 2007;9(6):R126. doi: 10.1186/ar2339. [PubMed: 18062805].

6. Fauci AS. Harrison's principles of internal medicine. 2. McGraw-Hill, Medical Publishing Division; 2008.

7. Leach MJ, Kumar S. The clinical effectiveness of Ginger (Zingiber officinale) in adults with osteoarthritis. Int J Evid Based Healthc. 2008;6(3):311-20. doi: 10.1111/j.1744-1609.2008.00106.x. [PubMed: 21631828].

8. RW S. Osteoarthritis. ; 2007.

9. Masui T, Hasegawa Y, Yamaguchi J, Kanoh T, Ishiguro N, Suzuki S. Increasing postural sway in rural-community-dwelling elderly persons with knee osteoarthritis. J Orthop Sci. 2006;11(4):353-8. doi: 10.1007/s00776-006-1034-9. [PubMed: 16897198].

10. Salavati M, Mazaheri M, Negahban H, Sohani SM, Ebrahimian MR, Ebrahimi I, et al. Validation of a Persian-version of Knee injury and Osteoarthritis Outcome Score (KOOS) in Iranians with knee injuries. Osteoarthritis Cartilage. 2008,16(10):1178-82. doi: 10.1016/j.joca.2008.03.004. [PubMed:18411065].

11. Zamani B, Ebadi S, Mousavi SGA, Bayat N. Assessment of the incidence of osteoarthritis and its risk factors in patients with knee join pain referring to out patient departments of kashan, 2004. Feyz J Kashan Univ Med Sci. 2006;10(3).

12. Wang C, de Pablo P, Chen X, Schmid C, McAlindon T. Acupuncture for pain relief in patients with rheumatoid arthritis: a systematic review. Arthritis Rheum. 2008;59(9):1249-56. doi:10.1002/art.24009. [PubMed: 18759255].

13. Haq SA, Davatchi F. Osteoarthritis of the knees in the COPCORD world. Int J Rheum Dis. 2011;14(2):122-9. doi: 10.1111/j.1756-185X.2011.01615.X. [PubMed: 21518310].

14. Muirden KD. Community Oriented Program for the Control of Rheumatic Diseases: studies of rheumatic diseases in the developing world. Curr Opin Rheumatol. 2005;17(2):153-6. [PubMed: 15711227].

15. Mathers C, Vos T, Stevenson C. The burden of disease and injury in Australia. Australian Institute of Health and Welfare; 1999.

16. Reichenbach S, Dieppe PA, Nuesch E, Williams S, Villiger PM, Juni $P$. Association of bone attrition with knee pain, stiffness and disability: a cross-sectional study. Ann Rheum Dis. 2011;70(2):293-8. doi: 10.1136/ard.2010.132985. [PubMed: 20870808].

17. Dingle JT. The effects of NSAID on the matrix of human articular cartilages. Z Rheumatol. 1999;58(3):125-9. [PubMed: 10441838].

18. Setty AR, Sigal LH. Herbal medications commonly used in the practice of rheumatology: mechanisms of action, efficacy, and side effects. Semin Arthritis Rheum. 2005;34(6):773-84. doi: 10.1016/j.semarthrit.2005.01.011. [PubMed: 15942912].

19. McManus J, Daggy B, Fakoukakis E, Wong L, Wang H, Fisher L, et al. What is Osteoarthritis?.; 2015 
20. Al-Suhaimi EA, Al-Riziza NA, Al-Essa RA. Physiological and therapeutical roles of ginger and turmeric on endocrine functions. Am J Chin Med. 2011;39(2):215-31. doi: 10.1142/S0192415X11008762. [PubMed: 21476200].

21. Bruno G. COX-2. ; 2009.

22. Rahnama P, Montazeri A, Huseini HF, Kianbakht S, Naseri M. Effect of Zingiber officinale R. rhizomes (ginger) on pain relief in primary dysmenorrhea: a placebo randomized trial. BMC Complement Altern Med. 2012;12:92. doi: 10.1186/1472-6882-12-92. [PubMed: 22781186].

23. Spice Exhibit. Spices: Exotic Flavours \& Medicines: Ginger 2007. Available from: http://unitproj.library.ucla.edu/biomed/spice/index.cfm.

24. Al-Achi A. A current look at ginger use 2009. Available from: http:// www.uspharmacist.com/oldformat.asp.

25. Schulick P. Ginger: common spice \& wonder drug. Herbal Free Press; 1996.

26. AA J. Ginger Spice of the sedative.J Women's Health. 2009;27:96-7.

27. Langner E, Greifenberg S, Gruenwald J. Ginger: history and use. Adv Ther. 1997;15(1):25-44.

28. Paramdeep G. Efficacy and tolerability of ginger (Zingiber officinale) in patients of osteoarthritis of knee. Indian J Physiol Pharmacol. 2013;57(2):177-83. [PubMed: 24617168].
29. Bee TA, Liew A. Dietary supplements used in osteoarthritis. Proceedings of Singapore Healthcare. .

30. Naderi Z, Mozaffari-Khosravi H, Dehghan A, Fallah Hosseini H, Nadjarzadeh A. The effect of ginger (zingiber officinale) powder supplement on pain in patients with knee osteoarthritis: a double-blind randomized clinical trial. SSUJ. 2013;20(5):657-67.

31. Altman RD, Marcussen KC. Effects of a ginger extract on knee pain in patients with osteoarthritis. Arthritis Rheum. 2001;44(11):2531-8. [PubMed: 11710709].

32. Khalvat A. Comparing the effects of ginger (Zingiber officinale) extract and ibuprofen on patients with osteoarthritis. Arch Iran Med. 2005;8(4):267-71.

33. Srivastava KC, Mustafa T. Ginger (Zingiber officinale) in rheumatism and musculoskeletal disorders. Med Hypotheses. 1992;39(4):342-8. [PubMed: 1494322].

34. Stone JH. In: Primer on the Rheumatic Diseases. Klippel JH, Stone JH, Crofford LJ, White PH, editors. ; 2008. pp. 235-9.Treatment of osteoarthritis.

35. Ramezani M, Nekozad N. Comparison between the effectiveness of glucosamine sulfate and zintoma on clinical improvement of knee osteoarthritis. Ebnesina. 2011;14(3):29-34. 